\title{
The Maximum Principle for Global Solutions of Stochastic Stackelberg Differential Games*
}

\author{
Alain Bensoussan ${ }^{\dagger \ddagger}$, Shaokuan Chen ${ }^{\dagger}$ and Suresh P. Sethi ${ }^{\dagger}$
}

October 30,2012

\begin{abstract}
This paper obtains the maximum principle for both stochastic (global) openloop and stochastic (global) closed-loop Stackelberg differential games. For the closed-loop case, we use the theory of controlled forward-backward stochastic differential equations to derive the maximum principle for the leader's optimal strategy. In the special case of the open-loop linear quadratic Stackelberg game, we consider the follower's Hamiltonian system as the leader's state equation, derive the related stochastic Riccati equation, and show the existence and uniqueness of the solution to the Riccati equation under appropriate assumptions. However, for the closed-loop linear quadratic Stackelberg game, we can write the related Riccati equation consisting of forward-backward stochastic differential equations, while leaving the existence of its solution as an open problem.
\end{abstract}

Keywords: Stackelberg differential game, maximum principle, forward-backward stochastic differential equation, Riccati equation.

\section{Introduction}

In 1934, H. von Stackelberg introduced a concept of a hierarchical solution for markets where some firms have power of domination over others [28]. This solution concept is now known as the Stackelberg equilibrium or the Stackelberg solution which, in the context of two-person nonzero-sum static games, involves players with asymmetric roles, one leading (called the leader) and the other following (called the follower). A Stackelberg game proceeds with the leader announcing his policy prior to the start of the game. With the knowledge of the leader's strategy, the follower chooses a policy so as to optimize his own performance index. The leader, anticipating the follower's optimal response, picks the policy which optimizes his performance index on the rational reaction curve of the follower, which together with the corresponding policy of the follower is known as the Stackelberg solution.

${ }^{*}$ The first author is supported by WCU (World Class University) program through the National Research Foundation of Korea funded by the Ministry of Education, Science and Technology (R31 - 20007) and by the Research Grants Council of HKSAR (PolyU 5001/11P). The second author is supported by NNSF of China (Grant No.11101140).

†Naveen Jindal School of Management, The University of Texas at Dallas, Richardson, TX, USA.

${ }^{\ddagger}$ Graduate School of Business, The Hong Kong Polytechnic University, Hong Kong, and Graduate Department of Financial Engineering, Ajou University, Suwon, South Korea. 
In dynamic Stackelberg games, it becomes important to know the player's information sets at any given time. In this paper, we will consider two different information structures: i) open-loop for both players and ii) closed-loop perfect state (CLPS) for both players. Moreover, we will only treat global solution where the leader announces his entire strategy at the start of the game and the follower reacts to the entire strategy. The solutions of games with the first information structure will be termed (global) open-loop Stackelberg solutions, whereas the solutions of the games with the second information structure will be termed (global) closed-loop Stackelberg solutions. It is known that both these solutions suffer from time inconsistency, which results from the functional dependence of the follower's optimal response strategy on the leader's entire strategy on the duration of the game.

In addition to these concepts, there is another concept of feedback Stackelberg solution, where the Stackelberg property is retained at every stage (in the discrete-time setting) with the leader having only stagewise advantage over the follower. Since the continuoustime problem can be viewed as the number of stages becomes unbounded in any finite interval, stagewise advantage of the leader over the follower turns into instantaneous advantage. A good aspect of this solution is that it is time consistent. Readers interested in the theory and applications of this solution can refer to [2], [7], [10], [12], [13] and [14].

In an open-loop or closed-loop Stackelberg differential game, the follower aims at minimizing his cost functional in accordance with the leader's strategy on the whole duration of the game. Anticipating the follower's optimal response depending on his entire strategy, the leader chooses an optimal one in advance to minimize his own cost functional, based on the Hamiltonian system satisfied by the follower's optimal response. The difference between the two kinds of games is whether the information sets of the players involve the history of the state. The introduction of the history of the state in the closed-loop Stackelberg game, even in the deterministic case, makes it difficult to tackle, as the follower may not obtain his optimal response if the leader's announced strategy incorporates the memory of the state. Two approaches to circumvent this difficulty are introduced: the team approach and the maximum principle. For the former, one can refer to [1, [6] in the discrete-time setting and [19], 21], [22] and [4] in the continuous-time setting. For the latter, one can refer to [20] for nonclassical control problems arising from Stackelberg games. The idea of team approach is as follows: the leader first minimizes his cost functional over the controls of both the leader and the follower, yielding a lower bound on his cost functional and the team strategies for both players. Then the leader makes an effort to find a closed-loop strategy such that the follower's optimal response and the state trajectory will coincide with his team strategy and the team optimal trajectory, which leads to the lower bound on the leader's cost functional. The maximum principle approach restricts the leader's strategy to depend only on the initial state and the current state (memoryless perfect state information structure) and a nonclassical control problem faced by the leader is solved. It is worth noting that in this case, the follower's adjoint equation involves the derivative of the leader's strategy with respect to the state. Therefore, after incorporating the follower's adjoint variable as an augmented state, the leader encounters a nonclassical control problem with the feature that both the control and its derivative with respect to the state appear in the controlled forward-backward ordinary differential equation system. The authors provide two approaches to tackle this problem and give the necessary conditions satisfied by the leader's optimal strategy. One is to directly apply 
the variational technique to the state system with mixed-boundary conditions (the adjoint equation of the follower with a terminal condition). The other is to establish an equivalent relationship between such a nonclassical control problem and a classical control problem, which yields that the optimal strategy could be found in the space of affine functions. The phenomenon of time inconsistency is also analyzed by the authors. We will elaborate on the technical details and generalize their result to the stochastic setting in section 4 .

For the stochastic formulation of Stackelberg games involving white noise terms, Yong 30. studies the open-loop linear quadratic case, with control variables appearing in diffusion term of the state. To give a state feedback representation of the open-loop Stackelberg solution (in a non-anticipating way), the related Riccati equation is derived and sufficient conditions for the existence of its solution with deterministic coefficients are discussed. More recently, Øksendal et al [18] have considered a general stochastic open-loop Stackelberg differential game, proved a sufficient maximum principle, and applied the theory to continuous-time newsvendor problems.

In this paper, we study stochastic global Stackelberg differential games with openloop and closed-loop information structures. As we shall see, the problems confronted by the leader in both cases, from the current point of view, are control problems with the state equations being forward-backward stochastic differential equations (FBSDEs). The theories for nonlinear backward stochastic differential equations (BSDEs) and FBSDEs have been extensively studied over the last two decades following the initial work by Pardoux and Peng [23]. One can refer to, among others, [15], [16], [24], [26], [31], and the references therein, for the development of the theory of FBSDEs and their applications. With the help of the results in optimization problems for controlled FBSDEs (see, e.g., [27] and [32]), we obtain the maximum principle for the leader's optimal strategies in stochastic global Stackelberg games, and discuss linear quadratic problems as well as the corresponding Riccati equations.

This paper is organized as follows. In section 2 we formulate a stochastic Stackelberg game and give three types of concepts of equilibria. In section 3 we present the maximum principle for a stochastic open-loop Stackelberg game. In section 4 we focus on a stochastic closed-loop Stackelberg game and derive a maximum principle for the leader's optimal strategy. As examples, linear quadratic stochastic open-loop and closed-loop Stackelberg games are studied in section 5. For the open-loop linear quadratic case, we show the existence and uniqueness of the solution to the associated stochastic Riccati equation under some assumptions. For the closed-loop case, we simply derive a new Riccati equation consisting of FBSDEs, without investigating the issue of the existence of its solution.

\section{Problem formulation and definition of equilibria}

Let $(\Omega, \mathcal{F}, P)$ be a complete probability space on which is defined a $d$-dimensional standard Brownian motion $\{W(t), 0 \leq t \leq T\} .\left\{\mathcal{F}_{t}\right\}_{0 \leq t \leq T}$ is the natural filtration generated by $W$ and augmented by all the $P$-null sets in $\mathcal{F}$ and $\mathcal{P}$ is the predictable sub- $\sigma$-field of $\mathcal{B}([0, T]) \times \mathcal{F}$.

We consider a stochastic differential system

$$
\left\{\begin{aligned}
d x(t) & =f(t, x(t), u(t), v(t)) d t+\sigma(t, x(t)) d W(t), \\
x(0) & =x_{0}
\end{aligned}\right.
$$


where

$$
\begin{gathered}
f: \Omega \times[0, T] \times \mathbb{R}^{n} \times \mathbb{R}^{m_{1}} \times \mathbb{R}^{m_{2}} \rightarrow \mathbb{R}^{n}, \\
\sigma: \Omega \times[0, T] \times \mathbb{R}^{n} \rightarrow \mathbb{R}^{n \times d},
\end{gathered}
$$

are $\mathcal{P} \times \mathcal{B}\left(\mathbb{R}^{n+m_{1}+m_{2}}\right) / \mathcal{B}\left(\mathbb{R}^{n}\right)$ and $\mathcal{P} \times \mathcal{B}\left(\mathbb{R}^{n}\right) / \mathcal{B}\left(\mathbb{R}^{n \times d}\right)$ measurable, respectively, and $(u(\cdot), v(\cdot))$ are the decision variables of the leader and the follower, respectively. The cost functionals for the leader and the follower to minimize are described as follows

$$
\begin{aligned}
& J_{1}(u, v)=E\left[\int_{0}^{T} g_{1}(t, x(t), u(t), v(t)) d t+G_{1}(X(T))\right], \\
& J_{2}(u, v)=E\left[\int_{0}^{T} g_{2}(t, x(t), u(t), v(t)) d t+G_{2}(x(T))\right],
\end{aligned}
$$

with

$$
\begin{gathered}
g_{i}: \Omega \times[0, T] \times \mathbb{R}^{n} \times U \times V \rightarrow \mathbb{R}, \\
G_{i}: \Omega \times \mathbb{R}^{n} \rightarrow \mathbb{R},
\end{gathered}
$$

$i=1,2$, being $\mathcal{P} \times \mathcal{B}\left(\mathbb{R}^{n}\right) \times \mathcal{B}(U) \times \mathcal{B}(V) / \mathcal{B}(\mathbb{R})$ and $\mathcal{F}_{T} \times \mathcal{B}\left(\mathbb{R}^{n}\right) / \mathcal{B}(\mathbb{R})$ measurable, respectively.

According to the player's information sets at any given time, there are three types of Stackelberg games: (global) open-loop, (global) closed-loop, and feedback Stackelberg games.

Open-loop games: In an open-loop Stackelberg game, the leader's information set at time $t$ is $\left\{x_{0}, \mathcal{F}_{t}\right\}$. Therefore, the strategy $u$ announced by the leader is an $\mathcal{F}_{t}$-adapted process. The follower aims at minimizing his cost functional $J_{2}(u, v)$ in accordance with the leader's strategy $u$ on the whole duration of the game. His optimal response $\Phi(u)$ will be an adapted process such that

$$
J_{2}(u, \Phi(u)) \leq J_{2}(u, v), \quad \forall u, v .
$$

The leader, anticipating the follower's optimal response $\Phi$, picks the policy $u^{*}$ which optimizes his performance index on the rational reaction curve of the follower, i.e.,

$$
J_{1}\left(u^{*}, \Phi\left(u^{*}\right)\right) \leq J_{1}(u, \Phi(u)), \forall u .
$$

$\left(u^{*}, \Phi\left(u^{*}\right)\right)$ is a Stackelberg solution for an open-loop game.

Closed-loop games: In a closed-loop Stackelberg game, the information set for the leader at time $t$ is $\left\{\mathcal{F}_{t}, x_{s}, s \in[0, t]\right\}$ (closed-loop perfect state information). The strategy that the leader adopts now can incorporate the history information of the state. Since in general it is difficult for the follower to obtain his optimal response if the leader's announced strategy incorporates the whole history of the state, we only consider the closed-loop case under the memoryless perfect state information pattern, i.e., the information set of the leader at time $t$ is $\left\{x_{0}, x_{t}, \mathcal{F}_{t}\right\}$. For leader's each strategy $u\left(t, x_{0}, x\right)$, which is now a stochastic field, the follower tries to find his optimal response $\Psi(u)$ such that

$$
J_{2}(u, \Psi(u)) \leq J_{2}(u, v), \forall u, v .
$$

Taking into account the follower's optimal response, the leader should choose $u^{*}$ such that

$$
J_{1}\left(u^{*}, \Psi\left(u^{*}\right) \leq J_{1}(u, \Psi(u)), \forall u .\right.
$$


$\left(u^{*}, \Psi\left(u^{*}\right)\right)$ is a Stackelberg solution for a closed-loop game.

Feedback games: In a feedback Stackelberg game, the information set for the leader at time $t$ is $\left\{x_{t}, \mathcal{F}_{t}\right\}$ (feedback pattern). The significant mechanism difference between feedback games and the former two types of games is that the advantage of the leader over the follower in a feedback Stackelberg game is instantaneous not global, as the differential game could be viewed as the limit of the discrete-time game as the number of stages becomes unbounded (see [2]). Therefore, corresponding to the leader's instantaneous strategy $u(t, x)$, the follower will make an instantaneous response of the form $v(t, x, u(t, x))$, which depends on the current state and the leader's current action. A feedback solution is a pair of strategies $\left(u^{*}, v^{*}\right)$ such that

$$
\begin{aligned}
& J_{1}\left(u^{*}, v^{*}\left(u^{*}\right)\right) \leq J_{1}\left(u, v^{*}(u)\right), \forall u, \\
& J_{2}\left(u^{*}, v^{*}\left(u^{*}\right)\right) \leq J_{2}\left(u^{*}, v\left(u^{*}\right)\right) . \forall v .
\end{aligned}
$$

From the definition we can see that the feedback Stackelberg solution has some equilibrium feature, whereas the open-loop or closed-loop solution involves a sequential optimization at the level of the follower and the leader.

\section{Stochastic open-loop Stackelberg differential games}

We first introduce some notations. For two vectors $x$ and $y$ in $\mathbb{R}^{n},\langle x, y\rangle$ means the inner product $\sum_{i=1}^{n} x_{i} y_{i}$. For a function $f$ defined on $\mathbb{R}^{n}, D f$ or $\partial f$ means the gradient of $f$. Here we specify that throughout this paper all the vectors are column vectors and the gradient of a scalar function $f$ is $\frac{\partial f}{\partial x}=\left(\frac{\partial f}{\partial x_{1}}, \cdots, \frac{\partial f}{\partial x_{n}}\right)^{\top}$, while the gradient of a vector function $f=\left(f_{1}, \cdots, f_{m}\right)^{\top}$ is a matrix

$$
\frac{\partial f}{\partial x}=\left(\begin{array}{ccc}
\frac{\partial f_{1}}{\partial x_{1}} & \cdots & \frac{\partial f_{1}}{\partial x_{n}} \\
\vdots & \vdots & \vdots \\
\frac{\partial f_{m}}{\partial x_{1}} & \cdots & \frac{\partial f_{m}}{\partial x_{n}}
\end{array}\right) .
$$

We further introduce two spaces of adapted processes to be used in the definition of the solution to a FBSDE,

$$
\begin{aligned}
& \mathcal{S}^{2}\left(0, T ; \mathbb{R}^{n}\right):=\left\{\psi \mid \psi: \Omega \times[0, T] \rightarrow \mathbb{R}^{n}\right. \text { is a continous adapted process such that } \\
& \left.E \sup _{0 \leq t \leq T}|\psi(t)|^{2}<\infty\right\} \\
& \mathcal{M}^{2}\left(0, T ; \mathbb{R}^{n}\right):=\left\{\psi \mid \psi: \Omega \times[0, T] \rightarrow \mathbb{R}^{n}\right. \text { is an adapted process such that } \\
& \left.E \int_{0}^{T}|\psi(t)|^{2} d t<\infty\right\}
\end{aligned}
$$

And the above two spaces will be simply written as $\mathcal{S}^{2}$ and $\mathcal{M}^{2}$, respectively, if no confusion arises.

The admissible strategy spaces for the leader and the follower are denoted by

$$
\begin{aligned}
& \mathcal{U}=\left\{u \mid u: \Omega \times[0, T] \rightarrow U \text { is } \mathcal{F}_{t^{-}} \text {adapted and } E \int_{0}^{T}|u(t)|^{2} d t<+\infty\right\}, \\
& \mathcal{V}=\left\{v \mid v: \Omega \times[0, T] \rightarrow V \text { is } \mathcal{F}_{\left.t^{-} \text {adapted and } E \int_{0}^{T}|v(t)|^{2} d t<+\infty\right\}},\right.
\end{aligned}
$$


where $U$ and $V$ are subsets of $\mathbb{R}^{m_{1}}$ and $\mathbb{R}^{m_{2}}$.

For the completeness of this paper, we state the formulation of general stochastic openloop Stackelberg games and the corresponding maximum principle. From the definition in section 2, given the leader's strategy $u \in \mathcal{U}$, the follower is faced the stochastic control problem

subject to

$$
\min _{v \in \mathcal{V}} J_{2}(u, v)=E\left[\int_{0}^{T} g_{2}(t, x(t), u(t), v(t)) d t+G_{2}(x(T))\right]
$$

$$
\left\{\begin{aligned}
d x(t) & =f(t, x(t), u(t), v(t)) d t+\sigma(t, x(t)) d W(t), \\
x(0) & =x_{0} .
\end{aligned}\right.
$$

Suppose there exists a unique solution $v^{*}(u(\cdot)) \in \mathcal{V}$ to the above problem for each $u \in \mathcal{U}$. If we define

$$
H_{2}\left(t, x, u, v, p_{2}, q_{2}\right):=\left\langle p_{2}, f(t, x, u, v)\right\rangle+\left\langle q_{2}, \sigma(t, x)\right\rangle+g_{2}(t, x, u, v),
$$

then the maximum principle (see [33]) yields that there exists a pair of adapted processes $\left(p_{2}, q_{2}\right) \in \mathcal{S}^{2} \times \mathcal{M}^{2}$ such that

$$
\left\{\begin{aligned}
d x(t)= & f\left(t, x(t), u(t), v^{*}(t)\right) d t+\sigma(t, x(t)) d W(t), \\
-d p_{2}(t)= & \left\{\left(\frac{\partial f}{\partial x}\right)^{\top}\left(t, x(t), u(t), v^{*}(t)\right) p_{2}(t)+\left(\frac{\partial \sigma}{\partial x}\right)^{\top}(t, x(t)) q_{2}(t)\right. \\
& \left.+\frac{\partial g_{2}}{\partial x}\left(t, x(t), u(t), v^{*}(t)\right)\right\} d t-q_{2}(t) d W(t), \\
x(0)= & x_{0}, \quad p_{2}(T)=\frac{\partial G_{2}}{\partial x}(x(T)), \\
v^{*}(t)= & \arg \min _{v \in V} H_{2}\left(t, x(t), u(t), v, p_{2}(t), q_{2}(t)\right) .
\end{aligned}\right.
$$

We assume that by the last equation in (3.1) a function $v=v^{*}\left(t, x, u, p_{2}\right)$ is implicitly and uniquely defined. After substituting $v=v^{*}\left(t, x, u, p_{2}\right)$ into the follower's maximum principle, we get the control problem faced by the leader

$$
\min _{u \in \mathcal{U}} J_{1}(u)=E\left[\int_{0}^{T} g_{1}\left(t, x(t), u(t), v^{*}\left(t, x(t), u(t), p_{2}(t)\right)\right) d t+G_{1}(X(T))\right]
$$

subject to

$$
\left\{\begin{aligned}
d x(t)= & f\left(t, x(t), u(t), v^{*}\left(t, x(t), u(t), p_{2}(t)\right)\right) d t+\sigma(t, x(t)) d W(t), \\
-d p_{2}(t)= & \left\{\left(\frac{\partial f}{\partial x}\right)^{\top}\left(t, x(t), u(t), v^{*}\left(t, x(t), u(t), p_{2}(t)\right)\right) p_{2}(t)+\left(\frac{\partial \sigma}{\partial x}\right)^{\top}(t, x(t)) q_{2}(t)\right. \\
& \left.+\frac{\partial g_{2}}{\partial x}\left(t, x(t), u(t), v^{*}\left(t, x(t), u(t), p_{2}(t)\right)\right)\right\} d t-q_{2}(t) d W(t), \\
x(0)= & x_{0}, \quad p_{2}(T)=\frac{\partial G_{2}}{\partial x}(x(T)) .
\end{aligned}\right.
$$

We denote

$$
\begin{aligned}
& H_{1}\left(t, u, x, y, p_{1}, p_{2}, q_{1}, q_{2}\right) \\
= & \left\langle p_{1}, f\left(t, x, u, v^{*}\left(t, x, u, p_{2}\right)\right)\right\rangle+\left\langle q_{1}, \sigma(t, x)\right\rangle+g_{1}\left(t, x, u, v^{*}\left(t, x, u, p_{2}\right)\right) \\
& -\left\langle y,\left(\frac{\partial f}{\partial x}\right)^{\top}\left(t, x, u, v^{*}\left(t, x, u, p_{2}\right)\right) p_{2}+\left(\frac{\partial \sigma}{\partial x}\right)^{\top}(t, x) q_{2}+\frac{\partial g_{2}}{\partial x}\left(t, x, u, v^{*}\left(t, x, u, p_{2}\right)\right)\right\rangle .
\end{aligned}
$$


Suppose $u^{*}$ is an optimal strategy for the leader. Then the maximum principle for controlled forward-backward stochastic differential equations (see, e.g., [27] or [32]) yields that there exists a triple of adapted processes $\left(p_{1}, q_{1}, y\right)$ such that

$$
u^{*}(t)=\arg \min H_{1}\left(t, u, x(t), y(t), p_{1}(t), p_{2}(t), q_{1}(t), q_{2}(t)\right),
$$

and

$$
\left\{\begin{aligned}
d y(t)= & -\frac{\partial H_{1}}{\partial p_{2}} d t-\frac{\partial H_{1}}{\partial q_{2}} d W(t), \\
= & -\left\{\left(\frac{\partial f}{\partial v} \frac{\partial v^{*}}{\partial p_{2}}\right)^{\top} p_{1}-\frac{\partial f}{\partial x} y-\sum_{i=1}^{n} y_{i}\left(\frac{\partial v^{*}}{\partial p_{2}}\right)^{\top} \frac{\partial}{\partial v}\left(\frac{\partial f}{\partial x_{i}}\right)^{\top} p_{2}\right. \\
& \left.-\left(\frac{\partial^{2} g_{2}}{\partial x \partial v} \frac{\partial v^{*}}{\partial p_{2}}\right)^{\top} y+\left(\frac{\partial v^{*}}{\partial p_{2}}\right)^{\top} \frac{\partial g_{1}}{\partial v}\right\} d t-\frac{\partial \sigma}{\partial x} y d W(t), \\
d p_{1}(t)= & -\frac{\partial H_{1}}{\partial x} d t+q_{1} d W(t) \\
= & -\left\{\frac{\partial f}{\partial x}+\frac{\partial f}{\partial v} \frac{\partial v^{*}}{\partial x}+\left(\frac{\partial \sigma}{\partial x}\right)^{\top} q_{1}+\frac{\partial g_{1}}{\partial x}+\left(\frac{\partial v^{*}}{\partial x}\right)^{\top} \frac{\partial g_{1}}{\partial v}\right. \\
& -\sum_{i} y_{i}\left[\frac{\partial}{\partial x}\left(\frac{\partial f}{\partial x_{i}}\right)^{\top}+\left(\frac{\partial v^{*}}{\partial x}\right)^{\top} \frac{\partial}{\partial v}\left(\frac{\partial f}{\partial x_{i}}\right)^{\top}\right] p_{2} \\
& \left.-\sum_{i} y_{i} \frac{\partial}{\partial x}\left(\frac{\partial \sigma}{\partial x_{i}}\right)^{\top} q_{2}-\left(\frac{\partial^{2} g_{2}}{\partial x^{2}}+\frac{\partial^{2} g_{2}}{\partial x \partial v} \frac{\partial v^{*}}{\partial x}\right)^{\top} y\right\} d t+q_{1} d W(t), \\
y(0)= & 0, p_{1}(T)=-\frac{\partial^{2} G_{2}}{\partial x^{2}}(x(T)) y(T)+\frac{\partial G_{1}}{\partial x}(x(T)) .
\end{aligned}\right.
$$

\section{Stochastic closed-loop Stackelberg games}

In this section, we consider a stochastic closed-loop Stackelberg game which is a stochastic version of the paper [20]. The difference between open-loop Stackelberg games and closedloop Stackelberg games is that in the former case the leader's information set is the $\sigma$-field $\mathcal{F}_{t}$ generated by the Brownian motion $W$, whereas in the latter case the leader's information set involves both the $\sigma$-field $\mathcal{F}_{t}$ and the history of the state $x$. As stated in the introduction, the difficulty of studying closed-loop Stackelberg games arises from the fact that the reaction of the follower can not be determined explicitly if the leader's strategy depends on the whole history of the state (CLPS information structure). However, if the leader's strategy is restricted to be memoryless, i.e., only the current state is involved in the strategy, Papavassilopoulos and Cruz [20] provide an efficient way to solve such a problem. As demonstrated in [20], the derivative $\frac{\partial u}{\partial x}$ of the leader's strategy $u$ will appear in the follower's adjoint equation and further in the leader's augmented state equation, which makes the leader's control problem a nonclassical one.

\subsection{The deterministic case revisited}

Since we apply the approach in Papavassilopoulos and Cruz [20] to solve the stochastic version of closed-loop Stackelberg games, we fist elaborate their techniques in this sub- 
section. The state and the cost functionals for the leader and the follower are as follows

$$
\begin{gathered}
\left\{\begin{array}{l}
\dot{x}(t)=f(t, x(t), u(t), v(t)), \\
x(0)=x_{0},
\end{array}\right. \\
J_{1}(u, v)=\int_{0}^{T} g_{1}(t, x(t), u(t), v(t)) d t+G_{1}\left(x_{T}\right), \\
J_{2}(u, v)=\int_{0}^{T} g_{2}(t, x(t), u(t), v(t)) d t+G_{2}\left(x_{T}\right) .
\end{gathered}
$$

Given the leader's strategy $u(t, x)_{t \in[0, T]}$ (we omit to write the dependence on the initial state $x_{0}$ ) which is continuously differentiable in $x$, if the follower's optimal response is $v^{*}$, then according to the deterministic maximum principle, there exists a function $p$ such that

$$
\left\{\begin{array}{l}
\dot{x}=f\left(t, x, u, v^{*}\right) \\
-\dot{p}=\left(\frac{\partial f}{\partial x}+\frac{\partial f}{\partial u} \frac{\partial u}{\partial x}\right)^{\top} p+\frac{\partial g_{2}}{\partial x}+\left(\frac{\partial u}{\partial x}\right)^{\top} \frac{\partial g_{2}}{\partial u} \\
\frac{\partial g_{2}}{\partial v}+\frac{\partial f}{\partial v} p=0 \\
x(0)=x_{0}, p(T)=\frac{\partial G_{2}(x(T))}{\partial x} .
\end{array}\right.
$$

Suppose we can get the unique solution

$$
v=\varphi(t, x, p, u)
$$

from solving

$$
\frac{\partial g_{2}}{\partial v}+\frac{\partial f}{\partial v} p=0
$$

Then, after substituting the expression (4.4) into (4.3) and $J_{1}$, the leader will be faced with the following problem

$$
\min _{u} J_{1}(u)=\int_{0}^{T} g_{1}(t, x, u, \varphi(t, x, p, u)) d t+G_{1}\left(x_{T}\right)
$$

subject to

$$
\left\{\begin{aligned}
\dot{x} & =f(t, x, u, \varphi(t, x, p, u)), \\
-\dot{p} & =\left[\frac{\partial f}{\partial x}+\frac{\partial f}{\partial u} \frac{\partial u}{\partial x}\right]^{\top} p+\frac{\partial g_{2}}{\partial x}+\left(\frac{\partial u}{\partial x}\right)^{\top} \frac{\partial g_{2}}{\partial u}, \\
x(0) & =x_{0}, p(T)=\frac{\partial G_{2}(x(T))}{\partial x} .
\end{aligned}\right.
$$

Since the derivative $\frac{\partial u}{\partial x}$ of the control variable $u$ is involved in the adjoint equation (4.6), the above problem is a nonclassical one. The authors provide two approaches to overcome this difficulty. One is the direct application of variational techniques. The other one is more interesting, which reveals the relative independence of $u$ and $\frac{\partial u}{\partial x}$ and the time inconsistency property. To be more precise, with $\frac{\partial u}{\partial x}$ replaced by another new control variable $\tilde{u}$, they construct a new classical problem

$$
\min _{u, \tilde{u}} \tilde{J}_{1}(u)=\int_{0}^{T} g_{1}(t, x, u, \varphi(t, x, p, u)) d t+G_{1}\left(x_{T}\right)
$$


subject to

$$
\left\{\begin{aligned}
\dot{x} & =f(t, x, u, \varphi(t, x, p, u)), \\
-\dot{p} & =\left(\frac{\partial f}{\partial x}+\frac{\partial f}{\partial u} \tilde{u}\right)^{\top} p+\frac{\partial g_{2}}{\partial x}+(\tilde{u})^{\top} \frac{\partial g_{2}}{\partial u}, \\
x(0) & =x_{0}, p(T)=\frac{\partial G_{2}(x(T))}{\partial x}
\end{aligned}\right.
$$

and prove the equivalence of the above nonclassical problem (4.5)-(4.6) and the constructed classical problem (4.7)-(4.8) in the sense that they have the same optimal trajectory and costs. Indeed, if we denote by $J_{1}^{*}$ and $J_{2}^{*}$ the optimal values of problems (4.5)-(4.6) and (4.7)-(4.8), respectively, then $J_{1}^{*} \geq J_{2}^{*}$. On the other hand, suppose that $\left(u^{*}, \tilde{u}^{*}\right)$ is an optimal control for problem (4.7)-(4.8) and $x^{*}$ is the corresponding trajectory, then control

$$
\hat{u}(t, x):=\tilde{u}^{*}(t) x+u^{*}(t)-\tilde{u}^{*}(t) x^{*}(t)
$$

yields the same trajectory $x^{*}$ and thus the same cost in problem (4.5)-(4.6). Consequently, $J_{1}^{*}=J_{2}^{*}$ and $\hat{u}$ is an optimal control for the nonclassical problem (4.5)-(4.6). Therefore, one can substitute $\frac{\partial u}{\partial x}$ for $\tilde{u}$ in the maximum principle for the problem (4.7)-(4.8) and finally get the maximum principle for the nonclassical problem (4.5)-(4.6) faced by the leader.

Remark 4.1. Given the leader's strategy $u(t, x)_{t \in[0, T]}$, the follower can also solve the following Hamilton-Jacobi-Bellman equation

$$
\left\{\begin{array}{l}
\frac{\partial V_{2}}{\partial t}+\inf _{v \in \mathbb{R}^{n}}\left\{\left\langle\frac{\partial V_{2}}{\partial x}, f(t, x, u(t, x), v)\right\rangle+g_{2}(t, x, u(t, x), v)\right\}=0 \\
V_{2}(T, x)=G_{2}(x)
\end{array}\right.
$$

and obtain the optimal feedback strategy

$$
v^{*}(t, x)=\arg \inf _{v \in \mathbb{R}^{n}}\left\{\left\langle\frac{\partial V_{2}}{\partial x}, f(t, x, u(t, x), v)\right\rangle+g_{2}(t, x, u(t, x), v)\right\}
$$

However, since $V_{2}$ depends on the whole function $u(\cdot)$, it is impossible for the leader to employ dynamic programming to depict his optimal strategy. The maximum principle approach turns out to be more appropriate for closed-loop Stackelberg games.

\subsection{The stochastic case}

In this subsection we tackle closed-loop Stackelberg games in the stochastic context, with the same idea as [20]. After introducing a stochastic disturbance term in the state equation (4.1), the adjoint equation for the follower, which also acts as the state equation in the leader's problem, will be a BSDE rather than an ODE with a terminal condition. Therefore, the leader will end up with a control problem in which the state equation consists of a SDE and a BSDE, with the feature that both the control $u$ and its derivative $\frac{\partial u}{\partial x}$ are introduced in the controlled system. With the results on the maximum principle for control problems of FBSDEs, we present the necessary conditions for the leader's optimal strategy to satisfy in a closed-loop Stackelberg game. 
We first introduce the admissible strategy spaces for the leader and the follower $\mathcal{U}:=\left\{u: u: \Omega \times[0, T] \times \mathbb{R}^{n} \rightarrow U\right.$ is $\mathcal{F}_{t}$-adapted for any $x \in \mathbb{R}^{n}, u(t, x)$ is continuously differentible in $x$ for any $(\omega, t) \in \Omega \times[0, T]$, and the derivative $\frac{\partial u}{\partial x}$ is bounded $\}$, $\mathcal{V}:=\left\{v: v: \Omega \times[0, T] \times \mathbb{R}^{n} \rightarrow V\right.$ is $\mathcal{F}_{t^{-}}$adapted for any $\left.x \in \mathbb{R}^{n}\right\}$.

Then, given the leader's strategy $u(t, x)$, the follower's optimal response strategy $v^{*}(t, x)$ is a solution to the following classical optimal control problem,

$$
\min _{v \in \mathcal{V}} J_{2}=E \int_{0}^{T} g_{2}(t, x(t), u(t, x(t)), v(t)) d t+E G_{2}(X(T)),
$$

subject to

$$
\left\{\begin{aligned}
d x(t) & =f(t, x(t), u(t, x(t)), v(t)) d t+\sigma(t, x(t)) d W(t), \\
x(0) & =x_{0}
\end{aligned}\right.
$$

According to the maximum principle, there exists a pair of adapted processes $\left(p_{2}, q_{2}\right) \in$ $\mathcal{S}^{2} \times \mathcal{M}^{2}$ such that

$v^{*}(t, x(t))=\arg \min _{v \in V}\left\{\left\langle p_{2}(t), f(t, x(t), u(t, x(t)), v)\right\rangle+\left\langle q_{2}, \sigma(t, x)\right\rangle+g_{2}(t, x(t), u(t, x(t)), v)\right\}$,

and

$$
\left\{\begin{aligned}
d p_{2}(t)= & -\left[\left(\frac{\partial f}{\partial x}+\frac{\partial f}{\partial u} \frac{\partial u}{\partial x}\right)^{\top} p_{2}+\left(\frac{\partial \sigma}{\partial x}\right)^{\top} q_{2}\right. \\
& \left.+\frac{\partial g_{2}}{\partial x}+\left(\frac{\partial u}{\partial x}\right)^{\top} \frac{\partial g_{2}}{\partial u}\right] d t+q_{2}(t) d W(t), \\
p_{2}(T)= & \frac{\partial G_{2}}{\partial x}(x(T)),
\end{aligned}\right.
$$

where $x(\cdot)$ is the solution of (4.12) with policies $u(t, x)$ and $v^{*}(t, x)$. Suppose for any leader's strategy $u(t, x)$, there exists a unique strategy $v^{*}(t, x)$ for the follower that minimizes his cost functional $J_{2}$. We also suppose that (4.13) yields $v^{*}=\varphi\left(t, x, u, p_{2}\right)$. Then, taking into account the follower's optimal response, the leader will be confronted with the optimal control problem

$$
\min _{u \in \mathcal{U}} J_{1}=E \int_{0}^{T} g_{1}\left(t, x(t), u(t, x(t)), \varphi\left(t, x(t), u(t, x(t)), p_{2}(t)\right)\right) d t+E G_{1}(x(T))
$$

subject to

$$
\left\{\begin{aligned}
d x(t)= & f\left(t, x(t), u(t, x(t)), \varphi\left(t, x(t), u(t, x(t)), p_{2}(t)\right)\right) d t+\sigma(t, x(t)) d W(t), \\
d p_{2}(t)= & -\left[\left(\frac{\partial f}{\partial x}+\frac{\partial f}{\partial u} \frac{\partial u}{\partial x}\right)^{\top} p_{2}+\left(\frac{\partial \sigma}{\partial x}\right)^{\top} q_{2}\right. \\
& \left.+\frac{\partial g_{2}}{\partial x}+\left(\frac{\partial u}{\partial x}\right)^{\top} \frac{\partial g_{2}}{\partial u}\right] d t+q_{2}(t) d W(t), \\
x(0)= & x_{0}, \quad p_{2}(T)=\frac{\partial G_{2}}{\partial x}(x(T)) .
\end{aligned}\right.
$$


It can be seen that, after incorporating the follower's adjoint variable as an augmented state, the leader encounters a controlled FBSDE, which is the counterpart of (4.6) in the deterministic context. For the solvability of FBSDEs, one can refer to [15], [26], 24], [31], and the references therein. Here we assume that the leader's problem is well-posed, i.e., for each $u(\cdot) \in \mathcal{U}$, there exists a unique triple $\left(x, p_{2}, q_{2}\right) \in \mathcal{S}^{2} \times \mathcal{S}^{2} \times \mathcal{M}^{2}$ solving FBSDE (4.16). Since the derivative $\frac{\partial u}{\partial x}$ of the control variable $u$ is involved in the BSDE in (4.16), we apply the techniques in the deterministic case to relate the above nonclassical control problem to a classical one.

Consider the optimization problem of a controlled FBSDE

$$
\min _{u_{1}, u_{2}} J\left(u_{1}(\cdot), u_{2}(\cdot)\right)=E \int_{0}^{T} g_{1}\left(t, x(t), u_{1}(t), \varphi\left(t, x(t), u_{1}(t), p_{2}(t)\right)\right) d t+E G_{1}(x(T)),
$$

subject to

$$
\left\{\begin{aligned}
d x(t)= & f\left(t, x(t), u_{1}(t), \varphi\left(t, x(t), u_{1}(t), p_{2}(t)\right)\right) d t+\sigma(t, x(t)) d W(t), \\
d p_{2}(t)= & -\left[\left(\frac{\partial f}{\partial x}+\frac{\partial f}{\partial u} u_{2}\right)^{\top} p_{2}+\left(\frac{\partial \sigma}{\partial x}\right)^{\top} q_{2}\right. \\
& \left.+\frac{\partial g_{2}}{\partial x}+\left(u_{2}\right)^{\top} \frac{\partial g_{2}}{\partial u}\right] d t+q_{2}(t) d W(t), \\
x(0)= & x_{0}, p_{2}(T)=\frac{\partial G_{2}}{\partial x}(x(T)),
\end{aligned}\right.
$$

where $u_{1}$ and $u_{2}$ are adapted control variables with values in $U$ and some bounded subset in $\mathbb{R}^{m_{1} \times n}$, respectively. Again we assume the above problem is well-posed. Obviously, if we denote by $J_{1}^{*}$ and $J^{*}$ the optimal values of problems (4.15)-(4.16) and (4.17)-(4.18), respectively, then $J_{1}^{*} \geq J^{*}$. On the other hand, if $\left(u_{1}^{*}, u_{2}^{*}\right)$ is a solution to problem (4.17)(4.18) and $x^{*}$ is the corresponding optimal state trajectory, then we can construct an optimal control $u^{*}$ for problem (4.15)-(4.16) as follows

$$
u^{*}(t, x):=u_{2}^{*}(t) x+u_{1}^{*}(t)-u_{2}^{*}(t) x^{*}(t) .
$$

Therefore, $J_{1}^{*}=J^{*}$, which implies that if $u^{*}(t, x)$ is a solution to problem (4.15)-(4.16) and $x^{*}$ is the corresponding optimal state trajectory, then $\left(u^{*}\left(t, x^{*}(t)\right), \frac{\partial u^{*}}{\partial x}\left(t, x^{*}(t)\right)\right)$ is an optimal control for problem (4.17)-(4.18) and leads to the same optimal state trajectory $x^{*}$. Thus we can obtain the maximum principle for problem (4.15)-(4.16) faced by the leader by means of the necessary conditions satisfied by the optimal control for problem (4.17)-(4.18) (see, e.g., 27] or [32]). To this end, we define

$$
\begin{aligned}
& H_{1}\left(t, u_{1}, u_{2}, x, y, p_{1}, p_{2}, q_{1}, q_{2}\right) \\
= & \left\langle p_{1}, f\left(t, x, u_{1}, \varphi\left(t, x, u_{1}, p_{2}\right)\right)\right\rangle+\left\langle q_{1}, \sigma(t, x)\right\rangle-\left\langle y,\left(\frac{\partial f}{\partial x}+\frac{\partial f}{\partial u} u_{2}\right)^{\top} p_{2}\right. \\
& \left.+\left(\frac{\partial \sigma}{\partial x}\right)^{\top} q_{2}+\frac{\partial g_{2}}{\partial x}+\left(u_{2}\right)^{\top} \frac{\partial g_{2}}{\partial u}\right\rangle+g_{1}\left(t, x, u_{1}, \varphi\left(t, x, u_{1}, p_{2}\right)\right) .
\end{aligned}
$$

Theorem 4.1. Suppose $u^{*}(t, x)$ is a solution to the leader's problem (4.15)-(4.16). Then there exists a triple $\left(y, p_{1}, q_{1}\right)$ such that

$$
\begin{aligned}
& \left(u^{*}(t, x(t)), \frac{\partial u^{*}}{\partial x}(t, x(t))\right) \\
= & \arg _{\left(u^{1}, u^{2}\right)} \min H_{1}\left(t, u^{1}, u^{2}, x(t), y(t), p_{1}(t), p_{2}(t), q_{1}(t), q_{2}(t)\right)
\end{aligned}
$$


and

$$
\left\{\begin{aligned}
d y(t) & =-\frac{\partial H_{1}}{\partial p_{2}} d t-\frac{\partial H_{1}}{\partial q_{2}} d W(t) \\
d p_{1}(t) & =-\frac{\partial H_{1}}{\partial x} d t+q_{1}(t) d W(t) \\
y(0) & =0, \quad p_{1}(T)=-\frac{\partial^{2} G_{2}}{\partial x^{2}}(x(T)) y(T)+\frac{\partial G_{1}}{\partial x}(x(T))
\end{aligned}\right.
$$

where $\left(x, p_{2}, q_{2}\right)$ is the solution of state equation (4.16) with control $u^{*}(t, x)$, and $\frac{\partial H_{1}}{\partial p_{2}}, \frac{\partial H_{1}}{\partial q_{2}}$ and $\frac{\partial H_{1}}{\partial x}$ in (4.22) are evaluated at

$$
\left(t, u^{*}(t, x(t)), \frac{\partial u^{*}}{\partial x}(t, x(t)), x(t), y(t), p_{1}(t), p_{2}(t), q_{1}(t), q_{2}(t)\right) .
$$

Remark 4.2. If $u$ is independent of $x$, we conclude in comparison with the arguments in section 3 that the closed-loop Stackelberg solution is reduced to the open-loop Stackelberg solution and the maximum principles for both cases are identical.

\section{The linear quadratic Stackelberg games}

In this section we consider linear quadratic open-loop and closed-loop Stackelberg games. Yong derives the Riccati equation for the open-loop Stackelberg game in [30] where the weighting matrices of the state and controls in the cost functionals are assumed not necessarily positive definite, and controls are allowed to appear in the diffusion term. For the follower's problem, the author uses the solutions of the follower's Riccati equation and a BSDE to give the state feedback representation of the follower's optimal strategy (one can also refer to [33, Page 313] for a similar derivation of the state feedback representation for a linear quadratic stochastic control problem with deterministic coefficients). To be precise, the author assumes that the follower's adjoint variable $p_{2}$ in (5.3) has the affine form

$$
p_{2}=P x+\phi .
$$

Applying Itô's formula to $p_{2}$ and taking into account (5.1) and (5.3), one can get the follower's Riccati equation with respect to $P$ and a BSDE for $\phi$. Then the author views the above BSDE for $\phi$, which contains the solution of the follower's Riccati equation and the leader's adopted strategy, and the original state equation as the leader's controlled system and further derives the leader's Riccati equation. Under some assumptions the author also discusses the solvability of the Riccati equations for the case of deterministic coefficients. Here we consider the follower's Hamiltonian system (5.4) as the leader's controlled state equation and hence the state feedback representation of the Stackelberg solution can be obtained at the same time for the leader and the follower. As a result, the corresponding Riccati equation here is of different form from the one in [30]. Since we deal with the case without decision variables in the diffusion term, we also show, under some appropriate assumptions, the existence and uniqueness of the solution to the derived Riccati equation with stochastic coefficients by means of a linear transformation to the standard stochastic Riccati equation. For the linear quadratic closed-loop Stackelberg game, we will see that the Hamiltonian system for the leader is no longer linear, which 
prevents us from getting an exogenous Riccati equation if we proceed the same way as in the open-loop case. Instead, we assume that the forward variable $y$ is linear with respect to the original state $x$ and derive an exogenous FBSDE which plays the same role as the Riccati equation in open-loop case. Throughout this section we assume the coefficients $A, B_{i}, C, Q_{i}, R_{i}, G_{i}$ are adapted bounded matrices, $Q_{i}, R_{i}, G_{i}$ are symmetric and nonnegative, and $R_{i}$ are uniformly positive, $i=1,2$.

\subsection{The open-loop case}

The state equation and cost functionals are given as follows.

$$
\begin{gathered}
\left\{\begin{array}{c}
d x(t)=\left(A x+B_{1} u+B_{2} v\right) d t+C x d W(t), \\
x(0)=x_{0},
\end{array}\right. \\
J_{1}(u, v)=\frac{1}{2} E\left[\int_{0}^{T}\left(\left\langle Q_{1} x(t), x(t)\right\rangle+\left\langle R_{1} u(t), u(t)\right\rangle\right) d t+\left\langle G_{1} x(T), x(T)\right\rangle\right], \\
J_{2}(u, v)=\frac{1}{2} E\left[\int_{0}^{T}\left(\left\langle Q_{2} x(t), x(t)\right\rangle+\left\langle R_{2} v(t), v(t)\right\rangle\right) d t+\left\langle G_{2} x(T), x(T)\right\rangle\right] .
\end{gathered}
$$

Given leader's strategy $u \in \mathcal{U}$, it is well known that the follower's problem

$$
\min _{v \in \mathcal{V}} J_{2}(u, v)=\frac{1}{2} E\left[\int_{0}^{T}\left(\left\langle Q_{2} x(t), x(t)\right\rangle+\left\langle R_{2} v(t), v(t)\right\rangle\right) d t+\left\langle G_{2} x(T), x(T)\right\rangle\right]
$$

subject to

$$
\left\{\begin{aligned}
d x(t) & =\left(A x+B_{1} u+B_{2} v\right) d t+C x d W(t) \\
x(0) & =x_{0}
\end{aligned}\right.
$$

is a standard linear quadratic optimal control problem and the unique solution is

$$
v^{*}(t)=-R_{2}^{-1} B_{2}^{\top} p_{2}
$$

where $p_{2}$ is the first part of the solution $\left(p_{2}, q_{2}\right) \in \mathcal{S}^{2} \times \mathcal{M}^{2}$ to the adjoint equation

$$
\left\{\begin{aligned}
-d p_{2}(t) & =\left(A^{\top} p_{2}+C^{\top} q_{2}+Q_{2} x\right) d t-q_{2} d W(t) \\
p_{2}(T) & =G_{2} x(T) .
\end{aligned}\right.
$$

Then, the leader's problem is

$$
\min _{u \in \mathcal{U}} J_{1}(u)=\frac{1}{2} E\left[\int_{0}^{T}\left(\left\langle Q_{1} x(t), x(t)\right\rangle+\left\langle R_{1} u(t), u(t)\right\rangle\right) d t+\left\langle G_{1} x(T), x(T)\right\rangle\right]
$$

subject to (the Hamiltonian system of the follower)

$$
\left\{\begin{aligned}
d x(t) & =\left(A x+B_{1} u-B_{2} R_{2}^{-1} B_{2}^{\top} p_{2}\right) d t+C x d W(t), \\
-d p_{2}(t) & =\left(A^{\top} p_{2}+C^{\top} q_{2}+Q_{2} x\right) d t-q_{2} d W(t) \\
x(0) & =x_{0}, p_{2}(T)=G_{2} x(T)
\end{aligned}\right.
$$


The leader's problem is well-posed since for every $u \in \mathcal{U}$, the coefficients of the system (5.4) satisfy the monotonicity condition proposed by Peng and $\mathrm{Wu}$ [26], which yields the existence and uniqueness of the solution $\left(x, p_{2}, q_{2}\right)$ to the system (5.4). Moreover, by similar arguments of Tang [29], we can get the following estimate

$$
E \sup _{0 \leq t \leq T}\left|p_{2}(t)\right|^{2}+E \sup _{0 \leq t \leq T}|x(t)|^{2}+E \int_{0}^{T}\left|q_{2}(t)\right|^{2} d t \leq L\left(\left|x_{0}\right|^{2}+E \int_{0}^{T}|u(t)|^{2} d t\right),
$$

where $L$ is a positive constant. With this estimate, we can adopt relevant arguments for standard linear quadratic optimal control problems in [17] and get the fact that the leader's objective functional $J_{1}(u)$ is convex in $u$,

$$
\lim _{\|u\| \rightarrow \infty} J_{1}(u)=\infty
$$

and $J_{1}(u)$ is Fréchet differentiable over $\mathcal{U}$ with the representation

$$
\begin{aligned}
\left\langle J_{1}^{\prime}(u), w\right\rangle= & E \int_{0}^{T}\left(\left\langle Q_{1}(t) x\left(t ; x_{0}, u\right), x(t ; 0, w)\right\rangle+\left\langle R_{1}(t) u(t), w(t)\right\rangle\right) d t \\
& +\left\langle G_{1} x\left(T ; x_{0}, u\right), x(T ; 0, w)\right\rangle .
\end{aligned}
$$

Here we use $x\left(\cdot ; x_{0}, u\right)$ to represent the solution of (5.4) with initial state $x(0)=x_{0}$ and control $u$. As a conclusion of Proposition 2.1.2 in [11, we know that the leader has a unique optimal strategy $u^{*} \in \mathcal{U}$ which satisfies $J_{1}^{\prime}\left(u^{*}\right)=0$. Now we use dual representation to characterize the optimal strategy $u^{*}$.

Theorem 5.1. For each $u \in \mathcal{U}$, there exists a unique solution $\left(x, y, p_{1}, q_{1}, p_{2}, q_{2}\right)$ to the $F B S D E$

$$
\left\{\begin{aligned}
d x(t) & =\left(A x+B_{1} u-B_{2} R_{2}^{-1} B_{2}^{\top} p_{2}\right) d t+C x d W(t), \\
-d p_{2}(t) & =\left(A^{\top} p_{2}+C^{\top} q_{2}+Q_{2} x\right) d t-q_{2} d W(t), \\
d y(t) & =\left(A y+B_{2} R_{2}^{-1} B_{2}^{\top} p_{1}\right) d t+C y d W(t), \\
-d p_{1}(t) & =\left(A^{\top} p_{1}+C^{\top} q_{1}-Q_{2} y+Q_{1} x\right) d t-q_{1} d W(t), \\
x(0) & =x_{0}, y(0)=0, p_{1}(T)=-G_{2} y(T)+G_{1} x(T), p_{2}(T)=G_{2} x(T) .
\end{aligned}\right.
$$

The necessary and sufficient condition for u to be the leader's optimal strategy is

$$
u(t)=-R_{1}^{-1} B_{1} p_{1}(t) .
$$

Proof. It can be seen that the FBSDEs consisting of $\left(x, p_{2}, q_{2}\right)$ and $\left(y, p_{1}, q_{1}\right)$ are two decoupled systems. Therefore, for given $u \in \mathcal{U}$, we can first get the unique solution $\left(x, p_{2}, q_{2}\right)$ to the equation

$$
\left\{\begin{aligned}
d x(t) & =\left(A x+B_{1} u-B_{2} R_{2}^{-1} B_{2}^{\top} p_{2}\right) d t+C x d W(t), \\
-d p_{2}(t) & =\left(A^{\top} p_{2}+C^{\top} q_{2}+Q_{2} x\right) d t-q_{2} d W(t), \\
x(0) & =x_{0}, p_{2}(T)=G_{2} x(T) .
\end{aligned}\right.
$$


Let $\tilde{y}:=-y$. Then FBSDE consisting of $\left(y, p_{1}, q_{1}\right)$ in (5.7) can be converted into the following one

$$
\left\{\begin{aligned}
d \tilde{y}(t) & =\left(A \tilde{y}-B_{2} R_{2}^{-1} B_{2}^{\top} p_{1}\right) d t+C \tilde{y} d W(t), \\
-d p_{1}(t) & =\left(A^{\top} p_{1}+C^{\top} q_{1}+Q_{2} \tilde{y}+Q_{1} x\right) d t-q_{1} d W(t), \\
\tilde{y}(0) & =0, p_{1}(T)=G_{2} \tilde{y}(T)+G_{1} x(T) .
\end{aligned}\right.
$$

The coefficients in the above system also satisfy the monotonicity condition in [26]. So there exists a unique solution to (5.9), which also implies the existence and uniqueness of the solution $\left(x, y, p_{1}, q_{1}, p_{2}, q_{2}\right)$ to FBSDE (5.7). The necessary part comes directly from the maximum principle (3.4) and (3.5). Now we prove the sufficient part. Denote by

$$
\left(x\left(\cdot ; x_{0}, u\right), y\left(\cdot ; x_{0}, u\right), p_{1}\left(\cdot ; x_{0}, u\right), q_{1}\left(\cdot ; x_{0}, u\right), p_{2}\left(\cdot ; x_{0}, u\right), q_{2}\left(\cdot ; x_{0}, u\right)\right)
$$

and

$$
\left(x(\cdot ; 0, w), y(\cdot ; 0, w), p_{1}(\cdot ; 0, w), q_{1}(\cdot ; 0, w), p_{2}(\cdot ; 0, w), q_{2}(\cdot ; 0, w)\right)
$$

the solutions to the system of FBSDEs (5.7) with initial states and controls as $\left(x_{0}, u\right)$ and $(0, w)$, respectively. Using Itô's formula to compute

$$
\left\langle p_{1}\left(t ; x_{0}, u\right), x(t ; 0, w)\right\rangle+\left\langle p_{2}(t ; 0, w), y\left(t ; x_{0}, u\right)\right\rangle
$$

and taking the expectation, we can get

$$
\begin{aligned}
\left\langle J_{1}^{\prime}(u), w\right\rangle= & E\left\langle G_{1} x\left(T ; x_{0}, u\right), x(T ; 0, w)\right\rangle \\
& +E \int_{0}^{T}\left\langle Q_{1}(t) x\left(t ; x_{0}, u\right), x(t ; 0, w)\right\rangle+\left\langle R_{1}(t) u(t), w(t)\right\rangle d t \\
= & E \int_{0}^{T}\left\langle R_{1}(t) u(t)+B_{1}^{\top}(t) p_{1}\left(t ; x_{0}, u\right), w(t)\right\rangle d t .
\end{aligned}
$$

Obviously $u=-R_{1}^{-1} B_{1}^{\top} p_{1}$ makes $J_{1}^{\prime}(u)$ equal to zero, so it is an optimal strategy for the leader.

From the uniqueness of the optimal strategy, we also know that FBSDE

$$
\left\{\begin{aligned}
d x(t) & =\left(A x-B_{1} R_{1}^{-1} B_{1}^{\top} p_{1}-B_{2} R_{2}^{-1} B_{2}^{\top} p_{2}\right) d t+C x d W(t), \\
-d p_{2}(t) & =\left(A^{\top} p_{2}+C^{\top} q_{2}+Q_{2} x\right) d t-q_{2} d W(t), \\
d y(t) & =\left(A y+B_{2} R_{2}^{-1} B_{2}^{\top} p_{1}\right) d t+C y d W(t), \\
-d p_{1}(t) & =\left(A^{\top} p_{1}+C^{\top} q_{1}-Q_{2} y+Q_{1} x\right) d t-q_{1} d W(t), \\
x(0) & =x_{0}, y(0)=0, p_{1}(T)=-G_{2} y(T)+G_{1} x(T), p_{2}(T)=G_{2} x(T),
\end{aligned}\right.
$$

has a unique solution $\left(x, y, p_{1}, q_{1}, p_{2}, q_{2}\right)$. And the Stackelberg solution $\left(u^{*}, v^{*}\right)$ can be written as

$$
u^{*}=-R_{1}^{-1} B_{1}^{\top} p_{1}, \quad v^{*}=-R_{2}^{-1} B_{2}^{\top} p_{2} .
$$

In what follows we see $(x, y)$ as the state and derive the feedback representation of the Stackelberg solution $\left(u^{*}, v^{*}\right)$ in terms of $(x, y)$. We denote

$$
\hat{x}=\left(\begin{array}{c}
x \\
y
\end{array}\right), \hat{p}=\left(\begin{array}{c}
p_{1} \\
p_{2}
\end{array}\right), \hat{q}=\left(\begin{array}{l}
q_{1} \\
q_{2}
\end{array}\right),
$$


and

$$
\begin{aligned}
& \hat{A}=\left(\begin{array}{cc}
A & 0 \\
0 & A
\end{array}\right), \hat{B}=\left(\begin{array}{cc}
B_{1} R_{1}^{-1} B_{1}^{\top} & B_{2} R_{2}^{-1} B_{2}^{\top} \\
-B_{2} R_{2}^{-1} B_{2}^{\top} & 0
\end{array}\right), \hat{C}=\left(\begin{array}{cc}
C & 0 \\
0 & C
\end{array}\right), \\
& \hat{Q}=\left(\begin{array}{cc}
Q_{1} & -Q_{2} \\
Q_{2} & 0
\end{array}\right), \hat{G}=\left(\begin{array}{cc}
G_{1} & -G_{2} \\
G_{2} & 0
\end{array}\right) .
\end{aligned}
$$

Then FBSDE (5.11) can be rewritten as

$$
\left\{\begin{array}{l}
d \hat{x}(t)=(\hat{A} \hat{x}(t)-\hat{B} \hat{p}(t)) d t+\hat{C} \hat{x} d W(t), \\
d \hat{p}(t)=-\left(\hat{A}^{\top} \hat{p}+\hat{C}^{\top} \hat{q}+\hat{Q} \hat{x}\right) d t+\hat{q} d W(t), \\
\hat{x}(0)=0, \hat{p}(T)=\hat{G} \hat{x}(T) .
\end{array}\right.
$$

Suppose there is a matrix-valued process $K$ such that

$$
\hat{p}=K \hat{x}
$$

and $K$ has a stochastic differential form

$$
d K(t)=M(t) d t+L(t) d W(t) .
$$

Applying Itô's formula to $K \hat{x}$, we get

$$
\begin{aligned}
& M \hat{x} d t+L \hat{x} d W(t)+K(\hat{A} \hat{x}-\hat{B} K \hat{x}(t)) d t+K \hat{C} \hat{x} d W(t)+L \hat{C} \hat{x} d t \\
= & d \hat{p}(t) \\
= & -\left(\hat{A}^{\top} K \hat{x}+\hat{C}^{\top} \hat{q}+\hat{Q} \hat{x}\right) d t+\hat{q} d W(t) .
\end{aligned}
$$

Comparing the diffusion terms in (5.16), we have

$$
\hat{q}=L \hat{x}+K \hat{C} \hat{x} .
$$

Substituting the expression into (5.16) and comparing the drift terms, we get

$$
\begin{aligned}
& M \hat{x}+K(\hat{A} \hat{x}-\hat{B} K \hat{x}(t))+L \hat{C} \hat{x} \\
= & -\hat{A}^{\top} K \hat{x}-\hat{C}^{\top}(L \hat{x}+K \hat{C} \hat{x})-\hat{Q} \hat{x},
\end{aligned}
$$

which yields

$$
M=-K \hat{A}-\hat{A}^{\top} K+K \hat{B} K-L \hat{C}-\hat{C}^{\top} L-\hat{C}^{\top} K \hat{C}-\hat{Q}
$$

Therefore, we get the Riccati equation

$$
\left\{\begin{array}{l}
d K(t)=-\left(K \hat{A}+\hat{A}^{\top} K-K \hat{B} K+L \hat{C}+\hat{C}^{\top} L+\hat{C}^{\top} K \hat{C}+\hat{Q}\right) d t+L d W(t), \\
K(T)=\hat{G}
\end{array}\right.
$$

The difference between the above Riccati equation and the standard one from stochastic LQ problems without control in diffusion terms (see, e.g., [25]) is that $\hat{B}, \hat{Q}$ and $\hat{G}$ here are not symmetric matrices. For $n=1$ and under some appropriate assumptions on the coefficient matrices, we show in the following proposition that Riccati equation (5.19) can be connected to a standard one through a linear transformation for FBSDE (5.13). 
Proposition 5.2. Suppose that $n=1$ and $\alpha$ and $\beta$ are two positive constants such that

$$
\frac{Q_{2}}{Q_{1}}=\frac{G_{2}}{G_{1}}=\alpha, \frac{B_{2} R_{2}^{-1} B_{2}^{\top}}{B_{1} R_{1}^{-1} B_{1}^{\top}}=\beta .
$$

Then, the Riccati equation (5.19) has a unique solution.

Proof. We make the transformation

$$
\hat{x}=\tilde{x}, \hat{p}=\Phi \tilde{p}, \hat{q}=\Phi \tilde{q},
$$

where

$$
\Phi=\left(\begin{array}{cc}
1 & -2 \beta \\
2 \alpha & 1
\end{array}\right) .
$$

Then FBSDE (5.13) can be converted into the following one

$$
\left\{\begin{array}{l}
d \tilde{x}(t)=(\tilde{A} \tilde{x}(t)-\tilde{B} \tilde{p}(t)) d t+\tilde{C} \tilde{x} d W(t), \\
d \tilde{p}(t)=-\left(\tilde{A}^{\top} \tilde{p}+\tilde{C}^{\top} \tilde{q}+\tilde{Q} \tilde{x}\right) d t+\tilde{q} d W(t), \\
\tilde{x}(0)=0, \tilde{p}(T)=\tilde{G} \tilde{x}(T),
\end{array}\right.
$$

where

$$
\begin{gathered}
\tilde{A}=\hat{A}, \tilde{C}=\hat{C} \\
\tilde{B}=\left(\begin{array}{cc}
B_{1} R_{1}^{-1} B_{1}^{\top}+2 \alpha B_{2} R_{2}^{-1} B_{2}^{\top} & -B_{2} R_{2}^{-1} B_{2}^{\top} \\
-B_{2} R_{2}^{-1} B_{2}^{\top} & 2 \beta B_{2} R_{2}^{-1} B_{2}^{\top}
\end{array}\right), \\
\tilde{Q}=\frac{1}{4 \alpha \beta}\left(\begin{array}{cc}
Q_{1}+2 \beta Q_{2} & -Q_{2} \\
-Q_{2} & 2 \alpha Q_{2}
\end{array}\right), \\
\tilde{G}=\left(\begin{array}{cc}
G_{1}+2 \beta G_{2} & -G_{2} \\
-G_{2} & 2 \alpha G_{2}
\end{array}\right) .
\end{gathered}
$$

Now the matrices $\tilde{B}, \tilde{Q}$ and $\tilde{G}$ are symmetric and positive definite. Suppose

$$
\tilde{p}=\tilde{K} \tilde{x},
$$

and

$$
d \tilde{K}=\tilde{K}_{1} d t+\tilde{L} d W(t) .
$$

With the same procedure to derive Riccati equation (5.19), we can get a standard Riccati equation for $(\tilde{K}, \tilde{L})$

$$
\left\{\begin{array}{l}
d \tilde{K}(t)=-\left(\tilde{K} \tilde{A}+\tilde{A}^{\top} \tilde{K}-\tilde{K} \tilde{B} \tilde{K}+\tilde{L} \tilde{C}+\tilde{C}^{\top} \tilde{L}+\tilde{C}^{\top} \tilde{K} \tilde{C}+\tilde{Q}\right) d t+\tilde{L} d W(t), \\
\tilde{K}(T)=\tilde{G}
\end{array}\right.
$$

According to the results in [8] or [25], or more general case in [29], we know that Riccati equation (5.22) has a unique solution $(\tilde{K}, \tilde{L})$ and

$$
\tilde{p}=\tilde{K} \tilde{x}, \tilde{q}=(\tilde{L}+\tilde{K} \tilde{C}) \tilde{x} .
$$


Consequently,

$$
\begin{aligned}
& \hat{p}=\Phi \tilde{p}=\Phi \tilde{K} \tilde{x}=\Phi \tilde{K} \hat{x}, \\
& \hat{q}=\Phi \tilde{q}=\Phi(\tilde{L}+\tilde{K} \tilde{C}) \tilde{x}=\Phi(\tilde{L}+\tilde{K} \tilde{C}) \hat{x} .
\end{aligned}
$$

Comparing (5.23) with (5.14) and (5.17), we finally get

$$
K=\Phi \tilde{K}, L=\Phi \tilde{L}
$$

From (5.12) we obtain that the Stackelberg solution $\left(u^{*}, v^{*}\right)$ has a feedback representation in terms of the state $(x, y)$.

\subsection{The closed-loop case}

As pointed out in the deterministic case [20], the relative independence of the leader's strategy $u$ and its derivative $\frac{\partial u}{\partial x}$ in a closed-loop Stackelberg game makes the leader so powerful that his Hamiltonian $H$ is likely to achieve $-\infty$ if there is no restriction on the derivative $\frac{\partial u}{\partial x}$. One way to restrict the leader's strength is to add a penalty term $\frac{\partial u}{\partial x}$ in his cost functional in order that $H$ is convex with respect to $\left(u, \frac{\partial u}{\partial x}\right)$. The other way is to impose a prior bounds on $\frac{\partial u}{\partial x}$ to retain $H$ finite. In this section we will adopt the latter way to assume $\frac{\partial u}{\partial x}$ to be bounded since it will appear as the coefficient of the unknowns in adjoint equations and the boundedness of the derivative $\frac{\partial u}{\partial x}$ implies the well-posedness of the leader's problem when affine strategies are adopted. For simplicity, we consider one-dimensional linear quadratic game, with the state equation and cost functionals of the two players as follows

$$
\left\{\begin{aligned}
d x(t) & =\left[A x(t)+B_{1} u(t)+B_{2} v(t)\right] d t+C x(t) d W(t), \\
x(0) & =x_{0}
\end{aligned}\right.
$$

and

$$
\begin{aligned}
& J_{1}=\frac{1}{2} E\left[\int_{0}^{T}\left(Q_{1} x^{2}(t)+R_{1} u^{2}(t)\right) d t+G_{1} x^{2}(T)\right], \\
& J_{2}=\frac{1}{2} E\left[\int_{0}^{T}\left(Q_{2} x^{2}(t)+R_{2} v^{2}(t)\right) d t+G_{2} x^{2}(T)\right] .
\end{aligned}
$$

The admissible strategy spaces from which the leader and the follower choose their strategies are given by

$$
\begin{aligned}
\mathcal{U}:= & \left\{u \mid u: \Omega \times[0, T] \times \mathbb{R} \rightarrow U \text { is } \mathcal{F}_{t^{-}} \text {adapted for any } x \in \mathbb{R}, u(t, x)\right. \text { is continuously } \\
& \text { differentible in } x \text { for any }(\omega, t) \in \Omega \times[0, T], \text { and the derivative }\left|\frac{\partial u}{\partial x}\right| \leq K \\
& \text { for some postive constant } K\}, \\
\mathcal{V}:= & \left\{v \mid v: \Omega \times[0, T] \times \mathbb{R}^{n} \rightarrow V \text { is } \mathcal{F}_{t^{-}} \text {adapted for any } x \in \mathbb{R}^{n}\right\} .
\end{aligned}
$$

Suppose for leader's each strategy $u \in \mathcal{U}$, the follower has a unique optimal response $v^{*} \in \mathcal{V}$. From (4.13) we know

$$
v^{*}=-R_{2}^{-1} B_{2} p_{2},
$$


with $p_{2}$ satisfying

$$
\left\{\begin{array}{l}
d p_{2}(t)=-\left[\left(A+B_{1} \frac{\partial u}{\partial x}\right) p_{2}+C q_{2}+Q_{2} x\right] d t+q_{2} d W(t), \\
p_{2}(T)=G_{2} x(T)
\end{array}\right.
$$

Therefore the leader's problem is

$$
\min _{u \in \mathcal{U}} J_{1}=\frac{1}{2} E\left[\int_{0}^{T}\left(Q_{1} x^{2}(t)+R_{1} u^{2}(t)\right) d t+G_{1} x^{2}(T)\right]
$$

subject to

$$
\left\{\begin{aligned}
d x(t) & =\left[A x(t)+B_{1} u(t, x(t))-R_{2}^{-1} B_{2}^{2} p_{2}(t)\right] d t+C x(t) d W(t), \\
d p_{2}(t) & =-\left[\left(A+B_{1} \frac{\partial u}{\partial x}\right) p_{2}+C q_{2}+Q_{2} x\right] d t+q_{2} d W(t), \\
x(0) & =x_{0}, p_{2}(T)=G_{2} x(T) .
\end{aligned}\right.
$$

Suppose that for every $u(t, x) \in \mathcal{U}$, there is a unique solution $\left(x, p_{2}, q_{2}\right)$ to $\operatorname{FBSDE}(\underline{5.25})$. According to the discussions in section 4.2, we know that the leader will lose nothing if he chooses his strategy among affine functions

$$
u(t, x)=u_{2}(t) x+u_{1}(t),
$$

with $u_{1}$ and $u_{2}$ being adapted processes and $\left|u_{2}\right| \leq K$. Then the leader's equivalent problem can be written as

$$
\min _{u_{1}, u_{2}} J_{1}=\frac{1}{2} E\left\{\int_{0}^{T}\left[Q_{1} x^{2}(t)+R_{1}\left(u_{2}(t) x(t)+u_{1}(t)\right)^{2}\right] d t+G_{1} x^{2}(T)\right\}
$$

subject to

$$
\left\{\begin{aligned}
d x(t) & =\left[\left(A+B_{1} u_{2}\right) x+B_{1} u_{1}-R_{2}^{-1} B_{2}^{2} p_{2}\right] d t+C x(t) d W(t), \\
d p_{2}(t) & =-\left[\left(A+B_{1} u_{2}\right) p_{2}+C q_{2}+Q_{2} x\right] d t+q_{2} d W(t), \\
x(0) & =x_{0}, p_{2}(T)=G_{2} x(T) .
\end{aligned}\right.
$$

For every pair $\left(u_{1}, u_{2}\right)$, the monotonicity condition guarantees the existence and uniqueness of the solution to (5.27). Therefore, the leader's problem with strategies restricted being of affine form is well-posed. In what follows we use the maximum principle to get the Hamiltonian system and related Riccati equation for leader's problem (5.26)-(5.27). Denote

$$
\begin{aligned}
& H_{1}\left(t, u_{1}, u_{2}, x, y, p_{1}, p_{2}, q_{1}, q_{2}\right) \\
= & p_{1}\left[\left(A+B_{1} u_{2}\right) x+B_{1} u_{1}-R_{2}^{-1} B_{2}^{2} p_{2}\right]+C x q_{1} \\
& -y\left[\left(A+B_{1} u_{2}\right) p_{2}+C q_{2}+Q_{2} x\right]+\frac{1}{2}\left[Q_{1} x^{2}+R_{1}\left(u_{2} x+u_{1}\right)^{2}\right] .
\end{aligned}
$$

To obtain $\left(u_{1}^{*}, u_{2}^{*}\right)$ that minimizes $H_{1}\left(t, u_{1}, u_{2}, x, y, p_{1}, p_{2}, q_{1}, q_{2}\right)$, we first fix $u_{2}$ and minimize $H_{1}$ with respect to $u_{1}$. By computation,

$$
u_{1}^{*}=-u_{2} x-R_{1}^{-1} B_{1} p_{1} .
$$


Substituting (5.29) into the expression (5.28) of $H$, we can see the only term containing $u_{2}$ is

$$
-B_{1} y p_{2} u_{2}
$$

Therefore, the optimal $u_{2}^{*}$ is

$$
u_{2}^{*}=\left\{\begin{array}{cl}
-K, & \text { if } \Delta>0 \\
K, & \text { if } \Delta<0 \\
\text { undefined, } & \text { if } \Delta=0
\end{array}\right.
$$

where

$$
\Delta:=-B_{1} y p_{2} .
$$

To find a candidate of optimal pair $\left(u_{1}^{*}, u_{2}^{*}\right)$, we set

$$
\begin{aligned}
u_{2}^{*} & :=\operatorname{bang}(K,-K ; \Delta) \\
& :=\operatorname{sgn}\left(B_{1} y p_{2}\right) K \\
& =\operatorname{sgn}(y) \operatorname{sgn}\left(B_{1} p_{2}\right) K \\
& =\operatorname{sgn}\left(p_{2}\right) \operatorname{sgn}\left(B_{1} y\right) K,
\end{aligned}
$$

where $\operatorname{sgn}$ is the sign function defined by

$$
\operatorname{sgn}(x)=\left\{\begin{array}{cl}
1 & \text { if } x>0 \\
0 & \text { if } x=0 \\
-1 & \text { if } x<0
\end{array}\right.
$$

From (5.29) we get

$$
u_{1}^{*}=-\operatorname{bang}(K,-K ; \Delta) x-R_{1}^{-1} B_{1} p_{1} .
$$

If $\left(u_{1}^{*}, u_{2}^{*}\right) \in \mathcal{U} \times \mathcal{V}$ is a solution to the leader's problem (5.26)-(5.27), then the maximum principle yields that there exist adapted processes $y, p_{1}$, and $q_{1}$ such that

$$
\left\{\begin{aligned}
d x(t) & =\left[\left(A+B_{1} u_{2}^{*}\right) x+B_{1} u_{1}^{*}-R_{2}^{-1} B_{2}^{2} p_{2}\right] d t+C x(t) d W(t), \\
d y(t) & =\left[\left(A+B_{1} u_{2}^{*}\right) y+R_{2}^{-1} B_{2}^{2} p_{1}\right] d t+C y d W(t), \\
d p_{1}(t) & =-\left[\left(A+B_{1} u_{2}^{*}\right) p_{1}+C q_{1}-Q_{2} y+Q_{1} x+R_{1} u_{2}^{*}\left(u_{2}^{*} x+u_{1}^{*}\right)\right] d t+q_{1} d W(t), \\
d p_{2}(t) & =-\left[\left(A+B_{1} u_{2}^{*}\right) p_{2}+C q_{2}+Q_{2} x\right] d t+q_{2} d W(t), \\
x(0) & =x_{0}, y(0)=0, p_{1}(T)=-G_{2} y(T)+G_{1} x(T), p_{2}(T)=G_{2} x(T), \\
u_{1}^{*} & =-\operatorname{bang}(K,-K ; \Delta) x-R_{1}^{-1} B_{1} p_{1}, u_{2}^{*}:=\operatorname{bang}(K,-K ; \Delta) .
\end{aligned}\right.
$$

Like the open-loop case, we proceed to express the optimal strategy $\left(u_{1}^{*}, u_{2}^{*}\right)$ in a nonanticipating way by means of the state feedback representation. Substituting the expressions of $u_{1}^{*}$ and $u_{2}^{*}$ into the FBSDE in (5.33), we get

$$
\left\{\begin{aligned}
d x(t) & =\left[A x-R_{1}^{-1} B_{1}^{2} p_{1}-R_{2}^{-1} B_{2}^{2} p_{2}\right] d t+C x(t) d W(t), \\
d y(t) & =\left[\left(A+B_{1} \operatorname{bang}(K,-K ; \Delta)\right) y+R_{2}^{-1} B_{2}^{2} p_{1}\right] d t+C y d W(t) \\
& =\left[A y+\operatorname{sgn}\left(p_{2}\right) K\left|B_{1} y\right|+R_{2}^{-1} B_{2}^{2} p_{1}\right] d t+C y d W(t), \\
d p_{1}(t) & =-\left[A p_{1}+C q_{1}-Q_{2} y+Q_{1} x\right] d t+q_{1} d W(t), \\
d p_{2}(t) & =-\left[\left(A+B_{1} \operatorname{bang}(K,-K ; \Delta)\right) p_{2}+C q_{2}+Q_{2} x\right] d t+q_{2} d W(t) \\
& =-\left[A p_{2}+\operatorname{sgn}(y) K\left|B_{1} p_{2}\right|+C q_{2}+Q_{2} x\right] d t+q_{2} d W(t), \\
x(0) & =x_{0}, y(0)=0, p_{1}(T)=-G_{2} y(T)+G_{1} x(T), p_{2}(T)=G_{2} x(T) .
\end{aligned}\right.
$$


In contrast to FBSDE (5.11) in the open-loop case, the presence of the additional nonlinear term $\operatorname{bang}(K,-K ; \Delta)$ in FBSDE (5.34) makes it a nonlinear system. Moreover, the Lipschitz continuity assumption usually made for the coefficients in the literature does not hold here. Therefore, the existence and uniqueness of the solution to (5.34), as far as we know, is still not available. On the other hand, if we still view $(x, y)$ as the "state" and represent $\left(p_{1}, p_{2}\right)$ in terms of $(x, y)$ as in the open-loop case, we can not derive an exogenous Riccati equation. Instead, we only see $x$ as the state and suppose

$$
y(t)=\xi(t) x(t), \quad p_{1}(t)=\eta(t) x(t), \quad p_{2}(t)=\zeta(t) x(t)
$$

and

$$
\begin{aligned}
& d \xi(t)=\xi_{1}(t) d t+\xi_{2}(t) d W(t) \\
& d \eta(t)=\eta_{1}(t) d t+\eta_{2}(t) d W(t) \\
& d \zeta(t)=\zeta_{1}(t) d t+\zeta_{2}(t) d W(t)
\end{aligned}
$$

By Itô's formula and in view of (5.35)

$$
\begin{aligned}
d y(t)= & \xi(t) d x(t)+x(t) d \xi(t)+C x(t) \xi_{2}(t) d t \\
= & \xi(t)\left[A x-R_{1}^{-1} B_{1}^{2} p_{1}-R_{2}^{-1} B_{2}^{2} p_{2}\right] d t+C \xi(t) x(t) d W(t) \\
& +\xi_{1}(t) x(t) d t+\xi_{2}(t) x(t) d W(t)+C \xi_{2}(t) x(t) d t \\
= & \left\{\left[A-R_{1}^{-1} B_{1}^{2} \eta(t)-R_{2}^{-1} B_{2}^{2} \zeta(t)\right] \xi(t)\right. \\
& \left.+\xi_{1}(t)+C \xi_{2}(t)\right\} x(t) d t+\left[C \xi(t)+\xi_{2}(t)\right] x(t) d W(t) .
\end{aligned}
$$

On the other hand,

$$
\begin{aligned}
d y(t) & =\left[\left(A+B_{1} \operatorname{bang}(K,-K ; \Delta)\right) y+R_{2}^{-1} B_{2}^{2} p_{1}\right] d t+C y d W(t) \\
& =\left[\left(A+B_{1} \operatorname{bang}(K,-K ; \tilde{\Delta})\right) \xi(t)+R_{2}^{-1} B_{2}^{2} \eta(t)\right] x(t) d t+C \xi(t) x(t) d W(t),
\end{aligned}
$$

where

$$
\tilde{\Delta}:=-B_{1} \xi(t) \zeta(t)
$$

Comparing (5.37) and (5.38), we have

$$
\begin{aligned}
& \xi_{2}(t)=0, \\
& \xi_{1}(t)=\left[R_{1}^{-1} B_{1}^{2} \eta(t)+R_{2}^{-1} B_{2}^{2} \zeta(t)+B_{1} \operatorname{bang}(K,-K ; \tilde{\Delta})\right] \xi(t)+R_{2}^{-1} B_{2}^{2} \eta(t) .
\end{aligned}
$$

With the same procedure, we can get

$$
\left\{\begin{array}{l}
\eta_{1}(t)=\left[R_{1}^{-1} B_{1}^{2} \eta(t)+R_{2}^{-1} B_{2}^{2} \zeta(t)-2 A-C^{2}\right] \eta(t)+Q_{2} \xi(t)-2 C \eta_{2}(t)-Q_{1}, \\
\zeta_{1}(t)=\left[R_{1}^{-1} B_{1}^{2} \eta(t)+R_{2}^{-1} B_{2}^{2} \zeta(t)-2 A-C^{2}-B_{1} \operatorname{bang}(K,-K ; \tilde{\Delta})\right] \zeta(t)-2 C \zeta_{2}(t)-Q_{2} .
\end{array}\right.
$$


Therefore, we derive the related Riccati equation for problem (5.26)-(5.27)

$$
\left\{\begin{aligned}
d \xi(t)= & \left\{\left[R_{1}^{-1} B_{1}^{2} \eta(t)+R_{2}^{-1} B_{2}^{2} \zeta(t)+B_{1} \operatorname{bang}(K,-K ; \tilde{\Delta})\right] \xi(t)+R_{2}^{-1} B_{2}^{2} \eta(t)\right\} d t \\
= & \left\{\left[R_{1}^{-1} B_{1}^{2} \eta(t)+R_{2}^{-1} B_{2}^{2} \zeta(t)\right] \xi(t)+\operatorname{sgn}(\zeta(t))\left|B_{1} \xi(t)\right|+R_{2}^{-1} B_{2}^{2} \eta(t)\right\} d t \\
d \eta(t)= & \left\{\left[R_{1}^{-1} B_{1}^{2} \eta(t)+R_{2}^{-1} B_{2}^{2} \zeta(t)-2 A-C^{2}\right] \eta(t)+Q_{2} \xi(t)-2 C \eta_{2}(t)\right. \\
& \left.-Q_{1}\right\} d t+\eta_{2}(t) d W(t), \\
d \zeta(t)= & \left\{\left[R_{1}^{-1} B_{1}^{2} \eta(t)+R_{2}^{-1} B_{2}^{2} \zeta(t)-2 A-C^{2}-B_{1} \operatorname{bang}(K,-K ; \tilde{\Delta})\right] \zeta(t)\right. \\
& \left.-2 C \zeta_{2}(t)-Q_{2}\right\} d t+\zeta_{2}(t) d W(t) \\
= & \left\{\left[R_{1}^{-1} B_{1}^{2} \eta(t)+R_{2}^{-1} B_{2}^{2} \zeta(t)-2 A-C^{2}\right] \zeta(t)-\operatorname{sgn}(\xi(t))\left|B_{1} \zeta(t)\right|\right. \\
& \left.-2 C \zeta_{2}(t)-Q_{2}\right\} d t+\zeta_{2}(t) d W(t), \\
\xi(0)= & 0, \eta(T)=-G_{2} \xi(T)+G_{1}, \zeta(T)=G_{2} .
\end{aligned}\right.
$$

Suppose $\left(\xi, \eta, \zeta, \eta_{2}, \zeta_{2}\right)$ is a solution to the above FBSDE and $x^{*}$ solves the linear SDE

$$
\left\{\begin{aligned}
d x(t) & =\left[A-R_{1}^{-1} B_{1}^{2} \eta-R_{2}^{-1} B_{2}^{2} \zeta\right] x(t) d t+C x(t) d W(t), \\
x(0) & =x_{0}
\end{aligned}\right.
$$

Then we can use Itô's formula to verify that

$$
\begin{aligned}
y(t) & :=\xi(t) x^{*}(t), p_{1}(t):=\eta(t) x^{*}(t), p_{2}(t):=\zeta(t) x^{*}(t), \\
q_{1}(t) & :=\left[C \eta(t)+\eta_{2}(t)\right] x^{*}(t), q_{2}(t):=\left[C \zeta(t)+\zeta_{2}(t)\right] x^{*}(t),
\end{aligned}
$$

together with $x^{*}$ solve the leader's Hamiltonian system (5.34). Therefore,

$$
u(t, x)=\operatorname{bang}(K,-K ; \tilde{\Delta}) x-\operatorname{bang}(K,-K ; \tilde{\Delta}) x^{*}(t)-R_{1}^{-1} B_{1} \eta(t) x^{*}(t)
$$

with $\tilde{\Delta}=-B_{1} \xi(t) \zeta(t)$ is a candidate of the leader's optimal strategy.

\section{References}

[1] T. Başar, "A new approach for derivation of closed-loop Stackelberg strategies," in Proceedings of the IEEE 17th Conference on Decision and Control, San Diego, CA, January 1979, IEEE Computer Society Press, Los Alamitos, CA, pp. 1113-1118.

[2] T. Başar and A. Haurie, "Feedback equilibria in differential games with structural and modal uncertainties," in Advances in Large Scale Systems (J. B. Cruz, Jr., Ed.), JAE Press Inc., Connecticut, 1984, pp. 163-201.

[3] T. Başar, A. Haurie and G. Ricci, "On the dominance of capitalists' leadership in a feedback Stackelberg solution of a differential game model of capitalism," J. Economic Dynamics and Control, 9:101-125, 1985.

[4] T. Başar and G. J. Olsder, "Team-optimal closed-loop Stackelberg strategies in hierarchical control problems," Automatica, 16(4):409-414, 1980.

[5] T. Başar and G. J. Olsder, Dynamic Noncooperative Game Theory, SIAM Series in Classics in Applied Mathematics, SIAM, Philadelphia PA, USA, 1999. 
[6] T. Başar and H. Selbuz, "Closed-loop Stackelberg strategies with applications in the optimal control of multilevel systems," IEEE Transactions on Automatic Control, 24(2):166-179, 1979.

[7] A. Bensoussan, S. Chen and S. P. Sethi, "Feedback Stackelberg solutions of infinitehorizon stochastic differential games," preprint.

[8] J. M. Bismut, "Controle des systems lineares quadratiques: applications de l'integrale stochastique," in Séminaire de Probabilités XII, Lecture Notes in Mathematics 649 (C. Dellacherie, P. A. Meyer, and M. Weil, Eds.), pp. 180-264, Springer-Verlag, Berlin, 1978.

[9] G. F. Cachon, "Supply chain coordination with contracts," in Handbooks in OR and MS Vol. 11, SCM: Design, Coordination and Cooperation (A. G. De Kok and S. C. Graves, Eds.), pp. 227-339, Elsevier, Amsterdam, The Netherlands, 2003.

[10] E. Dockner, S. Jøgensen, N. V. Long and G. Sorger, Differential Games in Economics and Management Science, Cambridge University Press, Cambridge, UK, 2000.

[11] I. Ekeland and R. Témam, "Convex Analysis and Variational Problems", NorthHolland, Amsterdam, 1976.

[12] X. He, A. Prasad and S. P. Sethi, "Cooperative advertising and pricing in a dynamic stochastic supply chain: feedback stackelberg strategies," Production and Operations Management, 18(1):78-94, 2009.

[13] X. He, A. Prasad, S. P. Sethi and G. J. Gutierrez, "A survey of Stackelberg differential game models in supply and marketing channels," Journal of Systems Science and Systems Engineering, 16(4):385-413, 2007.

[14] K. Kogan and C. S. Tapiero, "Supply Chain Games: Operations Management and Risk Valuation," Springer, New York, 2007.

[15] J. Ma, P. Protter and J. Yong, "Solving forward-backward stochastic differential equations explicitly-a four step scheme," Probability Theory and Related Fields, 98(3):339-359, 1994.

[16] J. Ma and J. Yong, "Forward-Backward Stochastic Differential Equations and their Applications," Lecture Notes in Mathematics (1702), Springer-Verlag, Berlin, 1999.

[17] Q. Meng, "General linear quadratic optimal stochastic control problem driven by a Brownian motion and a Poisson random martingale measure with random coefficients," arXiv:1102.3295v2.

[18] B. Øksendal, L. Sandal and J. Uboe, "Stochastic Stackelberg Equilibria with Applications to Time Dependent Newsvendor Models,",SSRN.

[19] G. P. Papavassilopoulos, "Leader-Follower and Nash Strategies with State Information," Ph. D. Dissertation, University of Illinois at Urbana-Champaign, August, 1979. 
[20] G. P. Papavassilopoulos and J. B. Cruz, Jr., "Nonclassical control problems and Stackelberg games," IEEE Transactions on Automatic Control, 24(2):155-166, 1979.

[21] G. P. Papavassilopoulos and J. B. Cruz, Jr., "Sufficient conditions for Stackelberg and Nash strategies with memory," Proc. Conference on Systems Engineering for Power: Organizational Forms for Large Scale Systems, Vol II, U. S. Dept. of Energy, Washington, DC, October, pp. 2.59-2.69, 1979.

[22] G. P. Papavassilopoulos and J. B. Cruz, Jr., "Sufficient conditions for Stackelberg and Nash strategies with memory," Journal of Optimization Theory and Applications, 31(2):233-260, 1980.

[23] E. Pardoux and S. Peng, "Adapted solution of a backward stochastic differential equation," Systems \& Control Letters, 14(1):55-61, 1990.

[24] E. Pardoux and S. Tang, "Forward-backward stochastic differential equations and quasilinear parabolic PDEs," Probability Theory and Related Fields, 114(2):123-150, 1999.

[25] S. Peng, "Stochastic Hamilton-Jacobi-Bellman equations", SIAM Journal on Control and Optimization, 30(2):284-304, 1992.

[26] S. Peng and Z. Wu, "Fully coupled forward-backward stochastic differential equations and applications to optimal control," SIAM Journal on Control and Optimization, 37(3):825-843, 1999.

[27] J. Shi and Z. Wu, "The maximum principle for fully coupled forward-backward stochastic control system," Acta Automatica Sinica, 32(2):161-169, 2006.

[28] H. von Stackelberg, Marktform und Gleichgewicht, Springer, Vienna, 1934. (An English translation appeared in The Theory of the Market Economy, Oxford University Press, Oxford, England, 1952.)

[29] S. Tang, "General linear quadratic optimal stochastic control problems with random coefficients: linear stochastic Hamilton systems and backward stochastic Riccati equations," SIAM Journal on Control and Optimization, 42(1):53-75, 2003.

[30] J. Yong, "A leader-follower stochastic linear quadratic differential game," SIAM Journal on Control and Optimization, 41(4):1015-1041, 2002.

[31] J. Yong, "Forward-backward stochastic differential equations with mixed initialterminal conditions," Trans. Amer. Math. Soc., 362(2):1047-1096, 2010.

[32] J. Yong, "Optimality Variational Principle for Controlled Forward-Backward Stochastic Differential Equations with Mixed Initial-Terminal Conditions," SIAM Journal on Control and Optimization, 48(6):4119-4156, 2010.

[33] J. Yong and X. Zhou, "Stochastic Controls: Hamiltonian Systems and HJB Equations," Springer, 1999. 